submitted to ApJ

\title{
The Effect of Poloidal Magnetic Field on Type I Planetary Migration: Significance of Magnetic Resonance
}

\author{
Takayuki Muto ${ }^{1}$, Masahiro N. Machida and Shu-ichiro Inutsuka \\ Department of Physics, Kyoto University, \\ Kitashirakawa-oiwake-cho, Sakyo-ku, Kyoto, 606-8502, Japan \\ muto@tap.scphys.kyoto-u.ac.jp
}

\begin{abstract}
We study the effect of poloidal magnetic field on type I planetary migration by linear perturbation analysis in the shearing-sheet approximation and the analytic results are compared with numerical calculations. In contrast to the unmagnetized case, the basic equations that describe the wake due to the planet in the disk allow magnetic resonances at which density perturbation diverges. In order to simplify the problem, we consider the case without magneto-rotational instability. We perform two sets of analyses: two-dimensional and three-dimensional. In twodimensional analysis, we find the generalization of the torque formula previously known in unmagnetized case. In three-dimensional calculations, we focus on the disk with very strong magnetic field and derive a new analytic formula for the torque exerted on the planet. We find that when Alfvén velocity is much larger than sound speed, two-dimensional torque is suppressed and three-dimensional modes dominate, in contrast to the unmagnetized case.
\end{abstract}

Subject headings: MHD — planets and satellites: formation — solar system: formation

\section{Introduction}

Type I planetary migration is one of serious difficulties in the theory of planet formation and there have been a lot of work on this topic. For an unmagnetized disk, extensive linear

\footnotetext{
${ }^{1}$ JSPS Research Fellow
} 
perturbation analyses have been performed since Goldreich \& Tremaine's pioneering work (Goldreich \& Tremaine, 1979). The recent result by Tanaka et al. (2002) has shown that the protoplanet of $5 \mathrm{M}_{\oplus}$ located at $5 \mathrm{AU}$ and embedded in the minimum-mass solar nebula (Hayashi et al. 1985) will migrate inward to the central star in $8 \times 10^{5}$ years, shorter than the observed time scale of protoplanetary disk, $10^{7}$ years (see e.g., Haisch et al. 2001). Their results are confirmed by numerical calculations (see e.g., D'Angelo et al. 2003).

Most of analytic works on type I planetary migration have focused on unmagnetized, locally isothermal disks. Goldreich \& Tremaine (1979) considered the two-dimensional disk, that is, there is no structure in the vertical direction of the disk, and derived analytic formulae of the torque exerted at Lindblad and corotation resonances. In order to calculate the migration rate, it is necessary to calculate the difference of the torque exerted on the disk inside and outside of the planet. This is done by Ward (1986). Later, Artymowicz (1993) derived, without invoking WKB approximation, a generalized formula for Lindblad resonances including the cutoff of the torque for high azimuthal mode number. Threedimensional analysis is performed by Takeuchi \& Miyama (1998), Tanaka et al. (2002), and Zhang \& Lai (2006). Takeuchi \& Miyama (1998) derived torque formulae for some resonances and full analytic calculation was performed by Zhang \& Lai (2006). When the planet is embedded in the equatorial plane of a thin disk, three-dimensional modes have been shown to be ineffective (Tanaka et al. 2002).

In contrast to all the above studies, magnetic fields are supposed to be present in protoplanetary disks. Significant mass accretion onto the central star requires an effective mechanism for angular momentum transfer. At present, magneto-rotational instability, or MRI (Balbus \& Hawley 1991), is the most likely mechanism for the generation of turbulent viscosity in the disks. However, whether the disk is magnetically active or dead depends strongly on the amount of gas (and hence, dust), number density, and the properties (size distribution etc.) of the dust grains because the surfaces of the dust grains are very efficient sites of recombination (Gammie 1996, Sano et al. 2000). The detailed analysis of the ionization structure with the standard cosmic ray ionization rate simply predicts that the planet forming region (approximately between $0.1 \mathrm{AU}$ and 10AU from the central star) might be in the dead zone where gas and magnetic field do not couple. However, various effects can change this prediction: the growth of dust grains to larger particles decreases the total surface area for recombination, and hence, increases the ionization degree. Sedimentation of dust grains onto the disk midplane also increases the ionization degree of most of the height of the disk. Yet another mechanism to increase the ionization rate is also proposed, which possibly removes the dead zone in the standard solar nebular model (Inutsuka \& Sano 2005).

The property of planetary migration may be totally different if magnetic field is impor- 
tant. Nelson and Papaloizou (2004) performed numerical calculations of type I migration in a magnetized disk and indicated that the turbulence due to MRI would result in stochastic torque on the planet. A simple model for the random torque was presented by Laughlin et al. (2004). Terquem (2003) performed the linear analysis of the torque for two-dimensional laminar disk with toroidal magnetic field and indicated that when stronger magnetic field was exerted on the disk inside the planet's orbit than the outside, inward migration might be halted. Fromang et al. (2005) further investigated this situation by numerical calculation and the numerical results showed good agreement with linear analysis.

In this paper, we investigate the type I planetary migration when the disk is exerted by poloidal magnetic field, which is a complementary analysis to Terquem (2003). As a first step to understand the nature of migration in a magnetized disk, we perform shearing sheet analysis, which assumes symmetric structure inside and outside the planet's orbit, and calculate the torque exerted on one side of the disk. We restrict ourselves to a laminar disk, the case without MRI, and derive analytic formulae of torque exerted on some important resonances. We perform a three-dimensional calculation and our formalism is a natural extension of previous studies of unmagnetized cases that is developed by Goldreich and Tremaine (1979) and Artymowicz (1993). For two-dimensional modes, we derive an analytic formula which generalizes that of Artymowicz (1993). For three-dimensional modes, we employ WKB approximation and derive an analytic torque formula in a strong field limit. We show, for three-dimensional modes, that there is a divergence in perturbed density at certain resonances and the torque is localized at this point. We describe how to treat the singularity in the wave equation. We show that two-dimensional modes are suppressed by poloidal magnetic field and three-dimensional modes will dominate the total torque. We then compare the results of the linear analysis with a numerical calculation, and show good agreement. Type I migration in a disk with strong poloidal magnetic field may be also important in the formation of planets around neutron stars (e.g., Bailes et al. 1991).

The plan of this paper is as follows. In section 2, we describe the linear analysis. In section 3, we describe the numerical calculation. Section 4 compares the results of linear analysis and numerical calculation. We discuss some possible important points that are not covered in this analysis in section [5, and section [6] is for our summary.

\section{Linear Calculation of Torque}

In this section, we derive analytic formulae for the torque exerted on the disk by the planet by linear analysis. The backreaction exerted on the planet causes orbital migration. 


\subsection{Basic Equations}

For simplicity, we consider only a local region around the planet using shearing sheet model (Naravan et al. 1987). Although it gives the same magnitude but the opposite sign of the torque between the inner and outer regions of the planet so the net torque becomes zero, we focus on one side of the disk in this setup and simplify the problem to understand the effect of magnetic field. We assume that the temperature is constant and the self-gravity of the disk is negligible in this local region. The orbit of the protoplanet is assumed to be circular on the equatorial plane of the disk. We set up local Cartesian coordinates with origin at the protoplanet's position and the $x^{-}, y$-, and $z$-axes are radial, azimuthal, and vertical direction of the disk, respectively. We use ideal MHD equations:

$$
\begin{gathered}
\frac{\partial \rho}{\partial t}+\nabla \cdot(\rho \boldsymbol{v})=0 \\
\frac{\partial \boldsymbol{v}}{\partial t}+(\boldsymbol{v} \cdot \nabla) \boldsymbol{v}=-\frac{1}{\rho} \nabla P-\nabla \psi_{\mathrm{eff}}-2 \Omega_{\mathrm{p}}\left(\boldsymbol{e}_{z} \times \boldsymbol{v}\right)-\frac{1}{4 \pi \rho} \boldsymbol{B} \times(\nabla \times \boldsymbol{B}) \\
\frac{\partial \boldsymbol{B}}{\partial t}=\nabla \times(\boldsymbol{v} \times \boldsymbol{B})
\end{gathered}
$$

where $\rho, \boldsymbol{v}, P, \psi_{\text {eff }}, \Omega_{\mathrm{p}}, \boldsymbol{e}_{z}$, and $B$ are the gas density, velocity, gas pressure, effective potential including tidal force and the planet's gravitational potential, Keplerian angular velocity of the protoplanet, a unit vector directed to the $z$-axis, and the magnetic flux density, respectively. We adopt an isothermal equation of state, $P=c^{2} \rho$, where $c$ is sound speed. The Keplerian angular velocity of the protoplanet is given by

$$
\Omega_{\mathrm{p}}=\left(\frac{G M_{\mathrm{c}}}{r_{\mathrm{p}}^{3}}\right)^{1 / 2}
$$

where $G, M_{\mathrm{c}}$, and $r_{\mathrm{p}}$ are the gravitational constant, mass of the central star, and the distance between the protoplanet and the central star, respectively. Our calculations are normalized by unit time, $\Omega_{\mathrm{p}}^{-1}$, unit velocity, $c$, and unit length, $h \equiv c / \Omega_{\mathrm{P}}$. The effective potential $\psi_{\text {eff }}$ in our normalization is given by, assuming a Keplerian disk,

$$
\tilde{\psi}_{\mathrm{eff}}=-\frac{3}{2} \tilde{x}^{2}-\frac{3 \tilde{r}_{\mathrm{H}}^{3}}{\tilde{r}}
$$

where all the quantities with tilde indicate the normalized value. The first term of the right hand side of equation (5) is composed of the gravitational potential of the central star and the centrifugal potential, and higher orders in $x, y$, and $z$ are neglected. We also neglect the $z$-dependence of the gravitational potential of the central star for simplicity, and consider later the constant background density. This greatly simplifies the calculation, and we have 
found that it does not seriously affect the results. The second term of the right hand side of equation (5) is the gravitational potential of the protoplanet, where $\tilde{r}_{\mathrm{H}}$ and $\tilde{r}$ are normalized Hill radius and the distance from the center of the protoplanet respectively. The Hill radius is defined by $r_{\mathrm{H}}=\left(M_{\mathrm{p}} / 3 M_{\mathrm{c}}\right)^{1 / 3} r_{\mathrm{p}}$, where $M_{\mathrm{p}}$ is the mass of the protoplanet.

The background disk is assumed to have no planet. The background gas flow has a Keplerian shear, $\boldsymbol{v}_{\mathbf{0}}=-(3 x / 2) \boldsymbol{e}_{y}$, background density is assumed to be constant, $\rho_{0}$, and the background magnetic field is assumed to be constant and poloidal, $\boldsymbol{B}_{0}=B_{0} \boldsymbol{e}_{z}$. We denote all the background quantities with subscript zero.

We treat the planet as a perturber on this background disk and derive the stationary pattern excited by the planet, as in Goldreich \& Tremaine (1979). We denote perturbed quantities with $\delta$, e.g., density perturbation is denoted as $\delta \rho$. We Fourier transform in $t-, y^{-}$, and $z$-directions, i.e., we shall consider the solution of the form $\delta \rho \propto \exp \left[-i\left(\omega t-k_{y} y-k_{z} z\right)\right]$. Since we consider the stationary pattern, the frequency $\omega$ is zero. The perturbed quantities are then

$$
\delta \rho(x, y, z)=\sum_{k_{y}, k_{z}} \delta \rho_{k_{y}, k_{z}}(x) e^{i\left(k_{y} y+k_{z} z\right)},
$$

and the inverse transformation is

$$
\delta \rho_{k_{y}, k_{z}}(x)=\frac{1}{L_{y} L_{z}} \int_{-L_{z} / 2}^{L_{z} / 2} \int_{-L_{y} / 2}^{L_{y} / 2} d y d z \delta \rho(x, y, z) e^{-i\left(k_{y} y+k_{z} z\right)},
$$

where $L_{y}$ and $L_{z}$ denote the box sizes of $y$ - and $z$-directions respectively. Imposing periodic boundary conditions in $y$ - and $z$-directions, the wave numbers in these directions are $k_{y}=$ $2 \pi n_{y} / L_{y}$ and $k_{z}=2 \pi n_{z} / L_{z}$ respectively, where $n_{y}$ and $n_{z}$ are integer. We shall drop the subscripts $k_{y}$ and $k_{z}$ of the Fourier modes unless it is ambiguous.

We define the Lagrangian displacement $\boldsymbol{\xi}$ by

$$
\begin{gathered}
\delta v_{x}=-i \sigma(x) \xi_{x} \\
\delta v_{y}=-i \sigma(x) \xi_{y}+\frac{3}{2} \Omega_{p} \xi_{x} \\
\delta v_{z}=-i \sigma(x) \xi_{z}
\end{gathered}
$$

where

$$
\sigma(x) \equiv \omega+\frac{3}{2} \Omega_{p} k_{y} x
$$

Using the Lagrangian displacement, the linearized induction equations are

$$
\begin{gathered}
\delta B_{x}=i k_{z} B_{0} \xi_{x} \\
\delta B_{y}=i k_{z} B_{0} \xi_{y} \\
\delta B_{z}=-B_{0}\left(\frac{d \xi_{x}}{d x}+i k_{y} \xi_{y}\right)
\end{gathered}
$$


The equation of continuity is

$$
\frac{\delta \rho}{\rho_{0}}+\frac{d \xi_{x}}{d x}+i k_{y} \xi_{y}+i k_{z} \xi_{z}=0
$$

and the equations of motion become, using the induction equations,

$$
\begin{gathered}
\left(-\sigma^{2}-3 \Omega_{p}^{2}\right) \xi_{x}+2 i \Omega_{p} \sigma \xi_{y}=-\left(c^{2}+v_{A}^{2}\right) \frac{d}{d x} \frac{\delta \rho}{\rho_{0}}+v_{A}^{2}\left(-k_{z}^{2} \xi_{x}-i k_{z} \frac{d \xi_{z}}{d x}\right)-\frac{d \psi_{p}}{d x} \\
-\sigma^{2} \xi_{y}-2 i \Omega_{p} \sigma \xi_{x}=-\left(c^{2}+v_{A}^{2}\right) i k_{y} \frac{\delta \rho}{\rho_{0}}-v_{A}^{2}\left(-k_{y} k_{z} \xi_{z}+k_{z}^{2} \xi_{y}\right)-i k_{y} \psi_{p} \\
-\sigma^{2} \xi_{z}=-c^{2} i k_{z} \frac{\delta \rho}{\rho_{0}}-i k_{z} \psi_{p}
\end{gathered}
$$

where $v_{A}^{2}=B_{0}^{2} / 4 \pi \rho_{0}$ denotes the Alfvén velocity of the background gas. We shall also define, for later convenience, the plasma $\beta$ by $c^{2} / v_{A}^{2}$.

The equations (15), (16), (17) and (18) are four independent equations for four variables

$\delta \rho$ and $\boldsymbol{\xi}$. The boundary conditions to be imposed are such that wave excited propagate away from the planet in both inner and outer parts of the disk.

Once the wave pattern is derived for each Fourier mode, $z$-component of the torque, of which backreaction causes the orbital migration, exerted on the disk by the planet for each mode is calculated by

$$
T_{k_{y}, k_{z}}=-2 L_{y} L_{z} \rho_{0} r_{p} k_{y} \int \operatorname{Im}\left(\frac{\delta \rho_{k_{y}, k_{z}}(x)}{\rho_{0}}\right) \psi_{\mathrm{pk}_{\mathrm{y}} \mathrm{k}_{\mathrm{z}}}(x) d x,
$$

where Im denotes the imaginary part.

\subsection{Wave Propagation Property of the Disk}

We shall investigate the wave propagation property of the disk with poloidal magnetic field. First, we derive a wave equation from (15)-(18). The $x$ - and $y$-components of the equations of motion can be written

$$
\begin{gathered}
\left(\sigma^{2}+3 \Omega_{p}^{2}-v_{A}^{2} k_{z}^{2}\right) \xi_{x}-2 i \Omega_{p} \sigma \xi_{y}=\frac{d f}{d x} \\
\left(\sigma^{2}-v_{A}^{2} k_{z}^{2}\right) \xi_{y}+2 i \Omega_{p} \sigma \xi_{x}=i k_{y} f
\end{gathered}
$$

where $f(x)$ is defined by

$$
f(x) \equiv \frac{1}{\sigma^{2}}\left[\left\{\left(c^{2}+v_{A}^{2}\right) \sigma^{2}-c^{2} v_{A}^{2} k_{z}^{2}\right\} \frac{\delta \rho}{\rho_{0}}+\left(\sigma^{2}-v_{A}^{2} k_{z}^{2}\right) \psi_{p}\right] .
$$


Equations (20) and (21) are the generalization of equation (10) of Goldreich \& Tremaine (1979). Variable $f(x)$ is related to the perturbation of total pressure $\delta \Pi=c^{2} \delta \rho+B_{0} \delta B_{z} / 4 \pi$ by

$$
f(x)=\frac{\delta \Pi}{\rho_{0}}+\psi_{\mathrm{p}}
$$

Therefore, it is a natural extension of the variable used by Goldreich \& Tremaine (1979) that is $c^{2} \delta \rho / \rho_{0}+\psi_{\mathrm{p}}$. Solving for $\xi_{x}$ and $\xi_{y}$,

$$
\begin{gathered}
\xi_{x}=\frac{1}{D}\left[\left(\sigma^{2}-v_{A}^{2} k_{z}^{2}\right) \frac{d f}{d x}-2 \Omega_{p} \sigma k_{y} f\right], \\
\xi_{y}=\frac{1}{D}\left[-2 i \Omega_{p} \sigma \frac{d f}{d x}+\left(\sigma^{2}+3 \Omega_{p}^{2}-v_{A}^{2} k_{z}^{2}\right) i k_{y} f\right],
\end{gathered}
$$

where $D$ is

$$
D=\left(\sigma^{2}-v_{A}^{2} k_{z}^{2}\right)\left(\sigma^{2}-v_{A}^{2} k_{z}^{2}+3 \Omega_{p}^{2}\right)-4 \sigma^{2} \Omega_{p}^{2} .
$$

This generalizes what is denoted by $D$ in the case of unmagnetized disk, e.g., equation (12) of Goldreich \& Tremaine (1979). Note that in the absence of magnetic field, $D$ is a quadratic function of $\sigma$, while this becomes a quartic function in the present situation. From equations (15) and (18), we finally obtain a second order ordinary differential equation which describes wave excitation and propagation of the disk,

$$
\frac{d^{2} f}{d x^{2}}+\mathcal{A}_{1} \frac{d f}{d x}+\mathcal{A}_{2} f=\mathcal{S}
$$

where

$$
\begin{gathered}
\mathcal{A}_{1}=\frac{d}{d x} \ln \frac{\sigma^{2}-v_{A}^{2} k_{z}^{2}}{D} \\
\mathcal{A}_{2}=\frac{\left(\sigma^{2}-c^{2} k_{z}^{2}\right) D}{\left\{\left(c^{2}+v_{A}^{2}\right) \sigma^{2}-c^{2} v_{A}^{2} k_{z}^{2}\right\}\left(\sigma^{2}-v_{A}^{2} k_{z}^{2}\right)}+\frac{2 \Omega_{p} \sigma k_{y}}{\sigma^{2}-v_{A}^{2} k_{z}^{2}} \frac{d}{d x}(\ln D)-k_{y}^{2}, \\
\mathcal{S}=\frac{\sigma^{2} D}{\left\{\left(c^{2}+v_{A}^{2}\right) \sigma^{2}-c^{2} v_{A}^{2} k_{z}^{2}\right\}\left(\sigma^{2}-v_{A}^{2} k_{z}^{2}\right)} \psi_{p} .
\end{gathered}
$$

Imposing WKB approximation, $d f / d x, k_{z} f \gg k_{y} f$, this equation simplifies to Schrödinger type:

$$
\frac{d^{2} f}{d x^{2}}+V(x) f=\mathcal{S}
$$

where

$$
V(x)=\frac{\left(\sigma^{2}-c^{2} k_{z}^{2}\right) D}{\left\{\left(c^{2}+v_{A}^{2}\right) \sigma^{2}-c^{2} v_{A}^{2} k_{z}^{2}\right\}\left(\sigma^{2}-v_{A}^{2} k_{z}^{2}\right)} .
$$

The regions where $V(x)>0$ are wave propagation regions, and those where $V(x)<0$ are evanescent. The boundary between these regions, where $V(x)=0$ or $V(x)= \pm \infty$, is 
the resonances. Figure 1 shows the appropriately normalized potential $V(x)$ for disk with $\beta=0.9$ and mode $k_{y} h=0.196$ and $k_{z} h=3.14$.

There are two or three points where $V(x)=0$ in one side of the disk with respect to the planet (either $x>0$ or $x<0$ ), depending on the value of $\beta$. From the condition $D=0$, we have

$$
\sigma^{2}=v_{A}^{2} k_{z}^{2}+\frac{1}{2}\left[\Omega_{p}^{2} \pm \sqrt{\Omega_{p}^{4}+16 \Omega_{p}^{2} v_{A}^{2} k_{z}^{2}}\right]
$$

We call the point with positive sign Lindblad Resonance Plus $(\mathrm{LR}+)$ and with negative sign Lindblad Resonance Minus (LR-). In the unmagnetized disk, LR+ coincides with the usual Lindblad resonance, $\sigma^{2}=\kappa^{2}$, where $\kappa$ is the epicycle frequency, and LR- degenerates into corotation point. We note that LR- exists only when $v_{A}^{2} k_{z}^{2}>3 \Omega_{p}^{2}$, which is exactly the same as the stability condition against MRI (Balbus \& Hawley 1991). When LR- does not exist but $k_{z} \neq 0$, the corotation region becomes a wave propagation region. Since $\sigma=0$ at the corotation, this indicates that there is a mode with zero frequency but non-zero wavelength, and therefore, in general, there is an unstable mode. Another condition for $V(x)=0$ is

$$
\sigma^{2}=c^{2} k_{z}^{2}
$$

This condition does not depend on magnetic field strength. This resonance corresponds to that found by Takeuchi \& Miyama (1998) and is named "Vertical Resonance" by Zhang \& Lai (2006). We shall also call this point Vertical Resonance (VR) in this paper.

There are two points in one side of the disk where $V(x)$ diverges. One is given by

$$
\sigma^{2}=v_{A}^{2} k_{z}^{2}
$$

At this point, the radial wavelength of Alfvén wave becomes zero. We shall call this point Alfvén Resonance (AR). Note that this divergence is related to what is called Alfvén resonance in plasma physics (see e.g., Stix 1992). The other point where $V(x)$ diverges is given by

$$
\sigma^{2}=\frac{c^{2} v_{A}^{2} k_{z}^{2}}{c^{2}+v_{A}^{2}} .
$$

At this point, the wavelength of the slow mode becomes zero, and this corresponds to "Magnetic Resonance" found by Terquem (2003) in the analysis of toroidal field. Therefore, we shall also call this point Magnetic Resonance (MR) in this paper.

For two-dimensional modes, $k_{z}=0$, only LR + exists, and the region in the vicinity of the corotation is evanescent region, whereas the regions further away from LR + are propagation regions. The details of the wave propagation property of the disk depend on the value of $\beta$ and $k_{z}$, but in general, there are three propagation regions on one side of the corotation, 
corresponding to the three wave modes of the magnetohydrodynamics. There are only two propagation regions in the analysis of Terquem (2003), since the mode is restricted to $k_{z}=0$ and the Alfvén wave with $\delta v_{z} \neq 0$ is not taken into account. Figure 2 shows the wave propagation property of one side of the disk in the case of $\beta<1$.

With careful investigation of the properties of the resonances, it is possible to analyze the wave excitation, propagation, and absorption. In this paper, however, we calculate the torque in some restricted cases and compare the results with numerical calculation. We first investigate the case when $k_{z}=0$. We then calculate three dimensional modes, $k_{z} \neq 0$, when very strong magnetic field is exerted. In these cases, MRI does not occur (Balbus \& Hawley 1991), and we expect the wave pattern becomes stationary with respect to the planet's motion.

\subsection{Two Dimensional Mode, $k_{z}=0$}

We consider the two-dimensional, or $k_{z}=0$, mode. In this case it is possible to calculate the torque exerted on the disk without imposing WKB approximation. When $k_{z}=0$, it is clear from equation (18) that the fluid particles do not move along $z$-axis, $\xi_{z}=0$, and the effect of magnetic field appears only in the pressure term. The sound speed becomes the phase velocity of the fast mode, $c^{2}+v_{A}^{2}$, and this acts as an effective sound speed. We can then follow the track of Artymowicz (1993) and calculate the torque exerted on the disk by evaluating the angular momentum flux carried by the wave at $|x| \rightarrow \infty$.

The position of the effective Lindblad resonance is given by

$$
\sigma^{2}\left(x_{\mathrm{eff}}\right)-\Omega_{p}^{2}-c^{2} k_{y}^{2}\left(1+\beta^{-1}\right)=0 .
$$

When

$$
\frac{\Omega_{p}^{2}+c^{2} k_{y}^{2}\left(1+\beta^{-1}\right)}{\Omega_{p} c k_{y} \sqrt{1+\beta^{-1}}} \gg 1
$$

the resonances inside and outside of the corotation radius are well isolated each other, and the torque exerted on the disk may be evaluated by the strength of the gravitational potential of the planet at the resonance point. The modified formula of the torque is

$$
T_{2 \mathrm{D}}=\frac{2 \pi}{3} r_{p} \rho_{0} L_{y} L_{z} \frac{\Omega_{p}}{\Omega_{p}^{2}+4 c^{2} k_{y}^{2}\left(1+\beta^{-1}\right)} \frac{1}{\sqrt{\Omega_{p}^{2}+c^{2} k_{y}^{2}\left(1+\beta^{-1}\right)}} \Psi_{\mathrm{eff}}^{2},
$$

where

$$
\Psi_{\mathrm{eff}}=\frac{d \psi_{p}}{d x}\left(x_{\mathrm{eff}}\right)-2 k_{y} \frac{\sqrt{\Omega_{p}^{2}+c^{2} k_{y}^{2}\left(1+\beta^{-1}\right)}}{\Omega_{p}} \psi_{p}\left(x_{\mathrm{eff}}\right)
$$


Details of the derivation are given in Appendix $\mathrm{A}$.

The equation (39) generalizes the equation (56) of Artymowicz (1993) to a magnetized disk. Since the effective sound speed becomes faster when poloidal magnetic fields present, the magnitude of the torque becomes smaller. There are two kinds of cutoff mechanism of the torque, as Artymowicz (1993) pointed out. One is mild cutoff that comes from the coefficient of $\Psi_{\text {eff }}$. The other is the sharp cutoff, which is the consequence of the fact that position of the effective Lindblad resonance goes further away from the corotation when magnetic field is stronger. In the case of planetary migration, the effect of sharp cutoff is more important, and the torque by two-dimensional mode is strongly suppressed when $\beta \lesssim 1$. We emphasize that we have obtained the torque formula (39) including the torque cutoff at high $k_{y}$ mode because we have not imposed WKB approximation, and hence, not neglected terms with $k_{y}$.

\subsection{Three Dimensional Mode, $k_{z} \neq 0$, in the Limit of Strong Magnetic Field}

In the unmagnetized disk, the contribution from three-dimensional, or $k_{z} \neq 0$, mode is small (Tanaka et al. 2002). However, it is indicated in the previous section that the twodimensional mode is strongly suppressed when strong magnetic field is present. Therefore three dimensional mode may be important in this case. We investigate three-dimensional mode in the limit of $\beta=0$. We impose WKB approximation in this section.

In the limit of $\beta \rightarrow 0$, the resonances LR + , LR-, and AR are infinitely far away from the corotation and we can safely neglect their contribution. The other two resonances, MR and VR, degenerate and the equation (27) becomes

$$
\frac{d^{2} f}{d x^{2}}-k_{z}^{2} f=S(x)
$$

where the source term $S(x)$ is

$$
S(x)=-\frac{\sigma^{2}}{\sigma^{2}-c^{2} k_{z}^{2}} \psi_{p} .
$$

In this case, waves are evanescent in the vicinity of the corotation, but there is a singularity in the source term at the degenerate point of MR and VR,

$$
\sigma_{\mathrm{MR}}^{2}=c^{2} k_{z}^{2} .
$$

The subscript MR denotes the quantities evaluated at this point. Note that this divergence originally comes from the divergence of the source term (30) at MR.

In order to regularize the singularity, we consider the small viscosity effective only in the vicinity of this point. The viscosity is effectively taken into account by adding the small 
positive imaginary part to the frequency,

$$
\sigma(x) \rightarrow \sigma(x)+i \epsilon
$$

where $\epsilon>0$ is small positive number (see e.g., Meyer-Vernet and Sicardy 1987). Taking the limit of $\epsilon \rightarrow 0$,

$$
\int \frac{g(x)}{\sigma-c k_{z}} d x=\mathcal{P} \int \frac{g(x)}{\sigma-c k_{z}} d x-i \pi \int \delta_{D}\left(\sigma-c k_{z}\right) g(x) d x
$$

where $g(x)$ is an arbitrary smooth function, $\mathcal{P}$ denotes the principal value of the integration and $\delta_{D}(x)$ is the Dirac's delta function.

The boundary condition we impose is that the perturbation must vanish for $|x| \rightarrow \infty$, i.e.

$$
\begin{aligned}
& f(x \rightarrow \infty) \propto e^{-k_{z} x} \\
& f(x \rightarrow-\infty) \propto e^{k_{z} x}
\end{aligned}
$$

The solution that satisfies this condition is

$$
f(x)=-\frac{1}{2 k_{z}}\left[e^{-k_{z} x} \int_{-\infty}^{x} e^{k_{z} u} S(u) d u-e^{k_{z} x} \int_{+\infty}^{x} e^{-k_{z} u} S(u) d u\right] .
$$

Substituting $S(x)$, the real and imaginary part of the solution are

$$
\begin{aligned}
\frac{2}{k_{z}} \operatorname{Re} f & =\mathcal{P} \int_{-\infty}^{x} e^{k_{z}(u-x)} \psi_{p}(u)\left[1+\frac{c k_{z}}{2}\left(\frac{1}{\sigma(u)-c k_{z}}-\frac{1}{\sigma(u)+c k_{z}}\right)\right] d u \\
& -\mathcal{P} \int_{+\infty}^{x} e^{k_{z}(x-u)} \psi_{p}(u)\left[1+\frac{c k_{z}}{2}\left(\frac{1}{\sigma(u)-c k_{z}}-\frac{1}{\sigma(u)+c k_{z}}\right)\right] d u
\end{aligned}
$$

and

$$
-\frac{6 \Omega_{p} k_{y}}{\pi c k_{z}^{2} \psi_{p, \mathrm{MR}}} \operatorname{Im} f=\left\{\begin{array}{cc}
e^{k_{z} x}\left[\exp \left(-\frac{2 c k_{z}^{2}}{3 \Omega_{p} k_{y}}\right)-\exp \left(\frac{2 c k_{z}^{2}}{3 \Omega_{p} k_{y}}\right)\right] & x<-x_{\mathrm{MR}} \\
\exp \left[-\frac{2 c k_{z}^{2}}{3 \Omega_{p} k_{y}}\right]\left(e^{-k_{z} x}-e^{k_{z} x}\right) & -x_{\mathrm{MR}}<x<x_{\mathrm{MR}} \\
-e^{-k_{z} x}\left[\exp \left(-\frac{2 c k_{z}^{2}}{3 \Omega_{p} k_{y}}\right)-\exp \left(\frac{2 c k_{z}^{2}}{3 \Omega_{p} k_{y}}\right)\right] & x>x_{\mathrm{MR}}
\end{array}\right.
$$

In the limit of $\beta \rightarrow 0$, the density perturbation is given by

$$
\frac{\delta \rho}{\rho_{0}}=\frac{1}{\sigma^{2}-c^{2} k_{z}^{2}}\left[\frac{\sigma^{2}}{v_{A}^{2}} f+k_{z}^{2} \psi_{p}\right] .
$$


In order to calculate the torque, we need imaginary part of $\delta \rho / \rho_{0}$ which is given by

$$
\begin{aligned}
2 c k_{z} \operatorname{Im}\left[\frac{\delta \rho}{\rho_{0}}\right]=-\pi\left\{\delta_{D}\left(\sigma-c k_{z}\right)-\right. & \left.\delta_{D}\left(\sigma+c k_{z}\right)\right\}\left(\frac{\sigma^{2}}{v_{A}^{2}} \operatorname{Re} f+k_{z}^{2} \psi_{p}\right) \\
& +\frac{\sigma^{2}}{v_{A}^{2}}\left\{\frac{\mathcal{P}}{\sigma-c k_{z}}-\frac{\mathcal{P}}{\sigma+c k_{z}}\right\} \operatorname{Im} f .
\end{aligned}
$$

It is possible to show that $f$ does not diverge at the resonance (see Appendix $B$ for details), and we can neglect the term with $\sigma^{2} / v_{A}^{2}$ in right hand side of the equation (51) when magnetic field is strong enough. Quantitatively, we can neglect these terms when

$$
\frac{\sigma^{2}}{v_{A}^{2}} \frac{c k_{z}^{2}}{\Omega_{p} k_{y}} \ll k_{z}^{2}
$$

since $f \sim \mathcal{O}\left(c k_{z}^{2} \psi_{p} / \Omega_{p} k_{y}\right)$. Since $\sigma^{2} \sim c^{2} k_{z}^{2}$ in the vicinity of the resonance, we obtain

$$
\beta \ll \frac{\Omega_{p} k_{y}}{c k_{z}^{2}}
$$

When this condition is satisfied, the imaginary part of the density perturbation is

$$
\operatorname{Im}\left[\frac{\delta \rho}{\rho_{0}}\right] \sim-\frac{\pi k_{z}}{2 c}\left\{\delta_{D}\left(\sigma-c k_{z}\right)-\delta_{D}\left(\sigma+c k_{z}\right)\right\} \psi_{p} .
$$

The first delta function indicates the torque exerted on the outer disk, and the second inner disk. These torques are of the same magnitude but different in sign. The magnitude of the torque on one side of the disk is then, from (19),

$$
T_{\mathrm{MR}}=\frac{2 \pi}{3} L_{y} L_{z} \frac{\rho_{0} r_{p} k_{z}}{\Omega_{p} c} \psi_{p, \mathrm{MR}}^{2}
$$

\section{Numerical Calculation}

We have performed numerical calculations in order to investigate how well the equations (39) and (56) describe the realistic value of the torque. We have done two sets of runs. One is for a two-dimensional disk. The other is for a three-dimensional thick disk.

\subsection{Numerical Methods}

We adopt the nested grid method (see, e.g., Machida et al. 2005, Matsumoto \& Hanawa, 2003) to obtain high spatial resolution near the planet. Each level of rectangular grid has 
the same number of cells $(=64 \times 256)$ for $2 \mathrm{D}$ run, while $(=64 \times 256 \times 16)$ for $3 \mathrm{D}$ run. The cell width $\Delta s(l)$ depends on the grid level $l$. The cell width is reduced by half with increasing grid level $(l \rightarrow l+1)$. We use 4 grid levels $(l=1,2 \cdots 4)$ for $2 \mathrm{D}$ run and 5 levels for $3 \mathrm{D}$ run. The box size of the coarsest grid $l=1$ is chosen to $\left(L_{x}, L_{y}\right)=(64 h, 256 h)$ for $2 \mathrm{D}$ run and $\left(L_{x}, L_{y}, L_{z} / 2\right)=(64 h, 256 h, 16 h)$ for $3 \mathrm{D}$ run. Note that in $z$-direction, the simulation box extends from midplane to $z=L_{z} / 2$. The box size of the finest grid is $(x, y)=(2 h, 8 h)$ for $2 \mathrm{D}$ run and $(x, y, z)=(2 h, 8 h, h)$ for $3 \mathrm{D}$ run. The cell width of the coarsest grid is $\Delta s(1)=h$, while that of the finest grid has $\Delta s(4)=0.125 h$ for $2 \mathrm{D}$ run and $\Delta s(5)=0.0625 h$ for $3 \mathrm{D}$ run. We assume the fixed boundary condition in the $x$-direction and periodic boundary condition in the $y$-direction. For $z$-direction, we impose a periodic boundary condition between $z=-L_{z} / 2$ and $z=L_{z} / 2$.

For two dimensional calculation, we neglect the $z$-dependence of the planet potential, i.e. we adopt the potential of the form

$$
\psi_{p}=-\frac{G M_{p}}{\sqrt{x^{2}+y^{2}}} .
$$

We employ the softening in the gravitational potential as follows. The gravitational force $\boldsymbol{F}$ by the planet is given by

$$
\boldsymbol{F}=\frac{G M_{p}}{(r+\varepsilon)^{3}} \boldsymbol{x},
$$

where $\varepsilon$ is the softening length, $r$ is the distance from the planet's position, and $\boldsymbol{x}$ is the position vector. We choose $\varepsilon$ such that this equals the mesh size of the finest grid, i.e., $\varepsilon=0.125 h$ for $2 \mathrm{D}$ run and $\varepsilon=0.0625 h$ for $3 \mathrm{D}$ run.

We fix the planet mass to be $\tilde{r}_{\mathrm{H}}=0.3$, corresponding to $3 M_{\oplus}$ planets when $M_{c}=M_{\odot}$ and $h / r_{p}=0.05$, and vary the initial strength of the poloidal magnetic field. We performed the calculations for $\beta=\infty, 100,10,2,0.3,0.1,0.01$, and 0.001 .

\section{Comparison between Numerical Calculation and Linear Analysis}

For all two-dimensional calculations and for three-dimensional calculation with $\beta=\infty$, 0.01 , and 0.001 , we do not observe MRI and steady states are realized. This is consistent with the stability criterion of MRI derived from linear analysis.

We then Fourier transform the density pattern of the steady state in $y$-and $z$-directions and calculate the torque exerted on one side of the disk by equation (19), and this torque is 
compared with the results of linear analysis. The normalization of the torque is taken to be

$$
\tilde{T}=\frac{T}{\rho_{0} r_{p} H^{4} \Omega_{p}^{2}} .
$$

We make use of FFT (e.g., Press et al. 1992). The wavenumber $k$ we evaluate is given by

$$
k=\frac{2 \pi n}{L}
$$

where $L$ is the box size of the $y$ - or $z$-directions and $n$ is an integer with $-N / 2<n<N / 2$ where $N$ is the mesh number. We also Fourier transform the gravitational potential of the planet numerically to obtain the value of $\psi_{p}$.

\subsection{Two-dimensional Calculation}

Figure 3 shows the stationary pattern of density perturbation obtained by two dimensional calculations for $\beta=0.01,2$, and 100. It is clear that, with increasing magnetic field, the amplitude of the wave becomes small and the point where waves are excited goes further away from the planet. Figure 4 shows the torque calculated as a result of numerical calculation for various magnetic field strength, or different $\beta$. It is clear that the torque becomes weaker as the magnetic field is stronger.

We show in figure 5 the comparison between the results of numerical calculation and linear analysis, equation (39). It is clear that for modes that satisfy condition (38), which we expect that equation (39) gives a good approximation for the torque, numerical calculation and linear analysis indeed show reasonably good agreement, at least an order of magnitude, even though equation (39) estimates the torque by the value of density perturbation only at the position of effective Lindblad resonance. Therefore, equation (39) is useful for estimating two-dimensional torque when poloidal magnetic field is exerted on the disk.

We also checked that the numerical calculation and linear analysis are in good agreement

for other values of $\beta$ except for $\beta=0.001$. For $\beta=0.001$, since the amplitude of density perturbation is very small, numerical torque is dominated by small noise in the disk.

\subsection{Three-dimensional Calculation}

For $\beta=0.01$ and $\beta=0.001$ models of the three-dimensional calculations, we do not observe MRI and steady state is realized. For other parameters, we observe the instability. 
Since we investigate the stationary pattern, we focus on results in which we do not observe MRI. We show in figure 6 the torque that is derived from numerical calculation for $n_{z}=$ 0,1 , and 2 , where $n_{z}$ is the mode number of $z$-direction. It is clear that $n_{z}=1$ modes overwhelm the two-dimensional modes in these models.

Figure 7 compares the torque calculated from the three-dimensional numerical calculations and that calculated from linear analysis of $n_{z}=1$ modes, torque formula (56). From the derivation of formula (56), this expression of the torque is valid when WKB condition

$k_{y} \ll k_{z}$ and strong magnetic field condition (54) are both satisfied. In the present parameter, the WKB condition is more restrictive. Since $k_{z} h=2 \pi / 32=0.196$ for $n_{z}=1$ mode, we expect that for $k_{y} h$ greater than this value, equation (56) does not give a good approximation for the torque. Nevertheless, the result of the numerical calculation indicates that the equation (56) shows a very good agreement even in the modes with $k_{y} h$ greater than this limit.

We also find that the imaginary part of the Fourier components of density perturbation diverges around MR, as expected from linear analysis. Figure 8 shows the profile of the imaginary part of the density perturbation of $\beta=0.001$ calculation for $\left(k_{y} h, k_{z} h\right)=(0.498,0.785)$. The position of magnetic resonance is indicated by an arrow. It is clear that density perturbation diverges at the resonance position and the contribution of the torque mostly comes from this divergence. The torque is localized at the magnetic resonances since waves cannot propagate on the disk, and, therefore, the analytic torque formula (56) gives a good approximation of the total torque, even if we consider the regions only in the vicinity of the resonance.

\section{Discussion}

\subsection{The Strength of Three-dimensional Modes in a Thin Disk}

Tanaka et al. (2002) has shown that in the unmagnetized disk, three-dimensional modes are subdominant. In contrast, when poloidal magnetic field is exerted on the disk, it is indicated that three-dimensional modes can dominate the torque when the magnetic field is sufficiently strong. In this section, we briefly discuss the critical value of $\beta$ at which $k_{z} \neq 0$ modes dominate the total torque in a thin magnetized disk according to the results of linear analysis. By "thin disk", we refer to the disk with small aspect ratio, smaller than that we have used in the numerical calculation, but not two-dimensional.

We calculate the torque for $n_{z}=0$ modes by (39) and $n_{z}=1$ modes by (56)). The Fourier transformation of the planet's gravitational potential is done numerically with the 
box size $L_{x}=32 h, L_{y}=128 h, L_{z} / 2=2 h$ and the spatial resolution $0.125 h$. We have checked that $n_{z}=2$ modes are smaller than $n_{z}=1$ modes. Figure 9 shows the torque for $\beta=0.01,2$, and 100. The vertical wavenumber of $n_{z}=1$ modes is $k_{z} h=1.57$, and WKB approximation is valid for $k_{y} h$ less than this value. For thin disk case, it is indicated, just as thick disk case, that two-dimensional modes are dominant for a weak magnetic field, while three-dimensional modes are important for a strong magnetic field case. We have investigated other values of $\beta$ and it is indicated that for the disk with $\beta \lesssim 0.1$, three-dimensional modes are more important than two-dimensional modes.

Since three-dimensional torque formula (56) is valid only in the strong magnetic field limit, it is not possible to extrapolate this to the case with $\beta \sim 1$. However, since the torque formula for two-dimensional modes (39) does not have any restriction, we can safely conclude that $k_{z}=0$ modes are always suppressed for strong magnetic field. Therefore, qualitatively, we expect that two-dimensional modes are suppressed for $\beta \lesssim 1$. To verify this conjecture quantitatively, we need careful analysis for $\beta \neq 0$ case, which will be presented elsewhere (T. Muto and S. Inutsuka 2008, in preparation).

\subsection{The Relation between Magnetic Field Strength and the Differential Torque}

For an unmagnetized disk, it is known that the outer torque that is exerted by the disk outside the planet wins over the inner torque exerted by the disk inside, when the disk gas density is larger in the inner disk than the outer (Ward 1997). This is the result of the competition of two effects. On one hand, since the inner density is larger than the outer, the inner torque becomes larger than the outer. On the other hand, the effect called pressure buffer enhances the outer torque. Considering the background disk structure, gas is slightly sub-Keplerian resulting from the outward pressure gradient. Since the planet is in Keplerian rotation, the corotation point locates slightly inside the planet, which makes the outer Lindblad resonance slightly closer to the planet. Calculating the difference of these two competing effects, the outer torque is larger than the inner torque.

Let us now qualitatively discuss the differential torque in the disk with poloidal magnetic field. First, we consider the two-dimensional mode when magnetic field exerted on the disk inside the planet's orbit is larger than the outside. In the case without variation in density and temperature, the mild cutoff of the torque by magnetic field makes the outer torque stronger. If the disk has radially decreasing magnetic pressure distribution, the planet locates slightly outside the corotation point since outward magnetic pressure is exerted on the gas. This also enhances the outer torque, since the outer effective Lindblad resonance locates 
closer to the planet. The outer torque is, therefore, expected to be stronger because of these two effects, in contrast to the toroidal magnetic field case of Terquem (2003) where the inner torque is stronger. This might indicate that the differential torque may be very sensitive to the configuration of magnetic field near the planet.

We now turn to the magnetic resonances of $k_{z} \neq 0$ modes, effective for low $\beta$. Since the formula (56) is for the limiting case of $\beta \rightarrow 0$, the torque does not depend on the magnetic field strength. We shall propose a simple torque formula for MR which generalizes equation (56) and discuss the effect of magnetic field. Firstly, we note that the relation between $f$ and $\delta \rho / \rho_{0}$ is given by

$$
\frac{\delta \rho}{\rho_{0}}=\frac{1}{\left(c^{2}+v_{A}^{2}\right) \sigma^{2}-c^{2} v_{A}^{2} k_{z}^{2}}\left[\sigma^{2} f-\left(\sigma^{2}-v_{A}^{2} k_{z}^{2}\right) \psi_{p}\right] .
$$

Since $f$ in the right hand side can be neglected when $\beta=0$, we expect this term can be neglected even in $\beta \neq 0$, provided that $\beta$ is sufficiently small. This equation also indicates that there is a $\delta$-function-like divergence at MR. Neglecting the term with $f$, we obtain the following torque formula at MR for low $\beta$,

$$
T_{\mathrm{MR}, \text { mod }}=\frac{2 \pi}{3} L_{y} L_{z} \frac{\rho_{0} r_{p} k_{z}}{\Omega_{p} c(1+\beta)^{3 / 2}} \psi_{p, \mathrm{MR}}^{2},
$$

where the value of gravitational potential is evaluated at MR. The smaller the magnetic field strength is, the closer towards the planet the MR position locates, which makes the gravitational potential at MR, $\psi_{\mathrm{p}, \mathrm{MR}}$, stronger. However, the coefficient, $(1+\beta)^{-3 / 2}$, becomes smaller, which makes the evaluation complicated. Using the parameters with threedimensional torque calculation, we evaluate the Fourier transform of the gravitational potential and calculate the torque. Figure 10 shows the torque calculated from the modified formula (62) for $\beta=0.001,0.01$, and 0.1. It is indicated that MR torque becomes smaller for weaker magnetic field strength. Therefore, we expect that when the inner magnetic field is stronger than the outer magnetic field and the field strength is high enough for $k_{z} \neq 0$ modes to be dominant, the inner torque wins over outer torque, in analogous to the results of the analysis of toroidal field by Terquem (2003).

When magnetic field is very strong, equation (62) indicates that the value of the torque is not sensitive to the strength of the field, and the differential torque can be very small. We consider, then, the effect of the gradient of sound speed, which changes the location of the resonance even in $\beta \rightarrow 0$ limit. Let us consider the disk with higher sound speed inside. The outer MR, which nearly degenerates with VR, is closer to the planet, giving a larger value of gravitational potential. The coefficient of the gravitational potential in equation (56) is inversely proportional to the sound speed because $L_{z} k_{z}$ is the mode number of the 
$z$-direction that is indifferent to the value of $c$. Therefore, the coefficient is smaller for the inner MR than the outer MR. The outer torque is expected to be stronger than the inner torque when magnetic field is very strong and when there is a negative gradient of the sound speed.

The qualitative dependence on magnetic field of the differential torque for laminar modes may be summarized as follows. Consider the case where inner magnetic field is stronger than the outer. When magnetic field strength is weak and two-dimensional modes are dominant, the outer torque is more enhanced and the migration is inward. When magnetic field is strong enough for three-dimensional modes to be dominant, the migration can be outward. Note, however, that the rate and directions of migration may depend sensitively on the gradient of the sound speed $c$. Negative gradient of the sound speed may cause the inward migration for very low $\beta$.

It seems difficult to halt the inward migration in a disk with strong poloidal magnetic field, since outward migration may require positive gradient of sound speed. Note, however, that the typical magnitude of one-sided torque is always smaller than the unmagnetized case, as shown in figure 9. In the disk with $\beta=100$, the torque is dominated by twodimensional modes and its magnitude is approximately $10^{-3}$ in our normalization, while in $\beta=0.01$ case, the magnitude is smaller by about two orders of magnitude. Therefore, the strong magnetization of disk is expected to slow down the migration. Actually this outcome is analogous to the effect of increasing gas temperature, and hence, thermal pressure and sound speed in the disk without magnetic field.

\subsection{Comparison with Toroidal Field Case}

In this paper, we have considered a protoplanetary disk threaded by a poloidal magnetic field. In the analysis of the torque at the magnetic resonance, we have considered a strong magnetic field case and derived a torque formula (56). We now consider briefly the more general case when toroidal component of magnetic field also exists. Although it is necessary to make a full, rigorous calculation including both poloidal and toroidal components of magnetic field in the background disk, we make a qualitative discussion by comparing the effect of magnetic resonances of purely toroidal and that of purely poloidal case.

In the case of toroidal magnetic field, there is a magnetic resonance in two-dimensional modes too (Terquem 2003). In the strong field limit, the position of the magnetic resonance is given by

$$
x_{\mathrm{MR}, \text { toroidal }}=\frac{2}{3} H .
$$


Since the magnetic resonance in poloidal case is located at

$$
x_{\mathrm{MR}, \text { poloidal }}=\frac{2}{3} \frac{k_{z}}{k_{y}} H
$$

and in a thin disk, modes $k_{z} \gg k_{y}$ are important, the magnetic resonance of a toroidal field is closer to the planet than the toroidal case. We also note that the Fourier components of threedimensional modes of gravitational potential are much smaller than the two-dimensional modes, as shown by Tanaka et al (2002). Therefore, in a standard case of a planet on a circular orbit embedded in a disk midplane, we expect that when the net magnetic field is dominated by toroidal components, the effect of poloidal magnetic field is small compared to that of a toroidal field. A possible exception is provided by a large inclination of planet's orbit. In this case, the gravitational potential of planet has a large z-component (threedimensional modes), and thus, the poloidal field would be important. Note, however, that we need a new set of analyses for the case of a planet with inclined orbit (for unmagnetized disks, see Tanaka \& Ward 2004).

\section{Summary}

We have performed linear perturbation analysis to calculate the torque exerted on the planet embedded in the non-turbulent disk with poloidal magnetic field using the shearing sheet approximation. We have derived a second order ordinary differential equation describing the excitation and propagation of the wave, equation (27), and derived analytic expressions of the torque for two limiting cases. Equation (39) gives the torque for twodimensional modes. Equation (56) gives the torque for three-dimensional modes for $\beta \ll 1$ under WKB approximation. We have compared the result of the linear analysis and numerical calculation, and found that both formulae show reasonable agreement. We have shown that the two-dimensional modes are suppressed when magnetic field is strong, in contrast to Terquem (2003) analysis of toroidal field, indicating that the property of planetary migration may be sensitive to the configuration of the magnetic field around the planet. It is also indicated that when magnetic field is very strong, three-dimensional modes are more effective than the two-dimensional modes, in contrast to the analysis of the three-dimensional calculation of unmagnetized disk by Tanaka et al. (2002)

Since we have been using the shearing sheet approximation and have derived the torque formulae only in restricted cases, the analysis of more general cases and other resonances is necessary, which will appear elsewhere (T. Muto and S. Inutsuka 2008, in preparation). Although the equation (56) agrees well with the numerical calculations, this is derived under

WKB approximation. Therefore, we need careful analysis for high $k_{y}$ for three-dimensional 
modes. We also need more quantitative analysis of the differential torque, which determines the direction and rate of the migration of the planet.

The authors thank T. Matsumoto for the great help in carrying out numerical calculations, and F. Masset and C. Terquem for useful discussions. This work is supported by the Grant-in-Aid for the 21st Century COE "Center for Diversity and Universality in Physics" from the Ministry of Education, Culture, Sports, Science and Technology (MEXT) of Japan. Numerical calculations were in part carried out on VPP5000 at the Center for Computational Astrophysics, CfCA, of National Astronomical Observatory of Japan. T. M. is supported by Grants-in-Aid for JSPS Fellows (19.2409) from MEXT of Japan. M. M. is supported by Grants-in-Aid (18740104) from MEXT of Japan. S. I. is supported by Grants-in-Aid (15740118, 16077202, and 18540238) from MEXT of Japan.

\section{A. Derivation of Torque Formula for $k_{z}=0$ Modes}

In this section, we derive the equation (39), following Artymowicz (1993) formalism. The linearized equations of continuity and motion for $k_{z}=0$ modes are

$$
\begin{gathered}
-i \sigma \frac{\delta \rho}{\rho_{0}}+\frac{d}{d x} \delta v_{x}+i k_{y} \delta v_{y}=0 \\
-i \sigma \delta v_{x}+c^{2}\left(1+\beta^{-1}\right) \frac{d}{d x} \frac{\delta \rho}{\rho_{0}}-2 \Omega_{p} \delta v_{y}=-\frac{d}{d x} \psi_{p}, \\
-i \sigma \delta v_{y}+\frac{1}{2} \Omega_{p} \delta v_{x}+i k_{y} c^{2}\left(1+\beta^{-1}\right) \frac{\delta \rho}{\rho_{0}}=-i k_{y} \psi_{p} .
\end{gathered}
$$

From these, we obtain the equation for vorticity:

$$
\frac{d}{d x} \delta v_{y}-\frac{1}{2} \Omega_{p} \frac{\delta \rho}{\rho_{0}}-i k_{y} \delta v_{x}=0 .
$$

Using the equations of motion, we finally obtain the Schrödinger-type second-order ordinary differential equation for $\delta v_{y}$ :

$$
\begin{array}{r}
\frac{d^{2}}{d x^{2}} \delta v_{y}+\frac{1}{c^{2}\left(1+\beta^{-1}\right)}\left[\sigma^{2}-\Omega_{p}^{2}-c^{2}\left(1+\beta^{-1}\right) k_{y}^{2}\right] \delta v_{y} \\
=\frac{1}{c^{2}\left(1+\beta^{-1}\right)}\left[-\frac{1}{2} \Omega_{p} \frac{d \psi_{p}}{d x}+\sigma k_{y} \psi_{p}\right]
\end{array}
$$

The position of the effective Lindblad resonance is given by

$$
\sigma^{2}=\Omega_{p}^{2}+c^{2}\left(1+\beta^{-1}\right) k_{y}^{2} .
$$


Waves are evanescent in the region in the vicinity of the planet and the regions further away from the resonance are propagation regions. Waves are excited at the effective Lindblad resonances and propagate away from the planet to $|x| \rightarrow \infty$.

We shall calculate the angular momentum flux at infinity. The angular momentum flux is calculated by

$$
F_{A}=2 r_{p} \rho_{0} L_{y} L_{z} \operatorname{Re}\left[\delta v_{x} \delta v_{y}^{*}\right] .
$$

Since the gravitational potential of the planet vanishes at infinity, the flux at the infinity is

$$
F_{A}(x \rightarrow \infty)=2 r_{p} \rho_{0} L_{y} L_{z} \frac{4 c^{2}\left(1+\beta^{-1}\right) k_{y}}{\Omega_{p}^{2}+c^{2}\left(1+\beta^{-1}\right) k_{y}^{2}} \operatorname{Im}\left[\delta v_{y}^{*} \frac{d}{d x} \delta v_{y}\right] .
$$

The solution of the wave equation (A5) is given by parabolic cylinder functions. Here, for simplicity, we assume the two resonances, inside and outside the planet, are isolated each other. The equation in the vicinity of the resonance is then given by

$$
\frac{d^{2}}{d z^{2}} \delta v_{y}+2 \gamma(z-\gamma) \delta v_{y}=-S
$$

where

$$
\begin{gathered}
z=\left[\frac{3 \Omega_{p} k_{y}}{2 c \sqrt{1+\beta^{-1}}}\right]^{\frac{1}{2}} x \\
\gamma^{2}=\frac{2}{3} \frac{\Omega_{p}^{2}+c^{2}\left(1+\beta^{-1}\right) k_{y}^{2}}{\Omega_{p} c k_{y} \sqrt{1+\beta^{-1}}} \\
S=\frac{\Omega_{p}}{c\left(1+\beta^{-1}\right)^{3 / 4} \sqrt{6 \Omega_{p} c k_{y}}}\left[\frac{d \psi_{p}}{d z}-\sigma\left(1+\beta^{-1}\right)^{1 / 4} \sqrt{\frac{8 c k_{y}}{3 \Omega_{p}^{3}}} \psi_{p}\right]_{\mathrm{eff}} .
\end{gathered}
$$

The subscript "eff" denotes the quantity evaluated at the resonance.

We impose the boundary condition as follows. In the evanescent region, the solution does not grow exponentially and in the propagation region, the waves propagate away from the planet. The solution is then

$$
\delta v_{y}=\frac{\pi S}{(2 \gamma)^{2 / 3}}\left\{\mathrm{Gi}\left[-(2 \gamma)^{1 / 3}(z-\gamma)\right]+i \mathrm{Ai}\left[-(2 \gamma)^{1 / 3}(z-\gamma)\right]\right\}
$$

Where Ai represents the Airy function and Gi is the solution of the equation (Abramowitz \& Stegun 1970)

$$
\frac{d^{2}}{d x^{2}} \mathrm{Gi}(x)-x \operatorname{Gi}(x)=-\frac{1}{\pi}
$$


Substituting the equation (A13) into (A8), we obtain the torque formula (39).

The condition of the isolation of the resonances is satisfied when the solution at one resonance does not affect the other resonance. The distance between the resonances is

$\delta z_{\text {res }} \sim \gamma$, while the scale that the solution in the vicinity of one resonance changes in the evanescent region is $\delta z_{\text {wave }} \sim \gamma^{-1 / 3}$. Hence, resonances are well separated each other when $\delta z_{\text {res }} \gg \delta z_{\text {wave }}$, or $\gamma \gg 1$.

\section{B. The Evaluation of the Magnitude of $f$}

In this section, we evaluate the integral in the equation (49). For simplicity, we set the planet's gravitational potential $\psi_{p}$ to be constant. The part of the equation (49),

$$
\int_{-\infty}^{x} d u e^{k_{z}(u-x)}-\int_{+\infty}^{x} d u e^{k_{z}(x-u)}
$$

is finite. We consider the rest. Since we are working on the local Cartesian coordinate where inside and outside the planet are symmetric, we assume $x>0$ without loss of generality. Let $I$ be

$$
\begin{aligned}
I & =\mathcal{P} \int_{-\infty}^{x} d u e^{k_{z}(u-x)}\left[\frac{1}{\sigma(u)-c k_{z}}-\frac{1}{\sigma(u)+c k_{z}}\right] \\
& -\mathcal{P} \int_{-\infty}^{x} d u e^{k_{z}(x-u)}\left[\frac{1}{\sigma(u)-c k_{z}}-\frac{1}{\sigma(u)+c k_{z}}\right],
\end{aligned}
$$

then

$$
\begin{gathered}
I=I_{A}-I_{B}-I_{C}+I_{D}, \\
I_{A}=\exp \left[-k_{z}\left(x-\frac{2 c k_{z}}{3 \Omega_{p} k_{y}}\right)\right] \operatorname{Ei}\left(k_{z} x-\frac{2 c k_{z}^{2}}{3 \Omega_{p} k_{y}}\right), \\
I_{B}=\exp \left[-k_{z}\left(x+\frac{2 c k_{z}}{3 \Omega_{p} k_{y}}\right)\right] \operatorname{Ei}\left(k_{z} x+\frac{2 c k_{z}^{2}}{3 \Omega_{p} k_{y}}\right), \\
I_{C}=\exp \left[k_{z}\left(x-\frac{2 c k_{z}}{3 \Omega_{p} k_{y}}\right)\right] \operatorname{Ei}\left(-k_{z} x+\frac{2 c k_{z}^{2}}{3 \Omega_{p} k_{y}}\right), \\
I_{D}=\exp \left[k_{z}\left(x+\frac{2 c k_{z}}{3 \Omega_{p} k_{y}}\right)\right] \operatorname{Ei}\left(-k_{z} x-\frac{2 c k_{z}^{2}}{3 \Omega_{p} k_{y}}\right),
\end{gathered}
$$

where Ei denotes the exponential integral,

$$
\operatorname{Ei}(w)=\mathcal{P} \int_{-\infty}^{w} d t \frac{e^{t}}{t}
$$


We can check $I_{A}, I_{B}, I_{C}$, and $I_{D}$ do not diverge at infinity by the asymptotic expansion of the exponential integral,

$$
e^{-w} \int_{-\infty}^{w} \frac{e^{t}}{t} d t \sim \frac{1}{w}
$$

and the inequality derived from the series expansion of the exponential integral,

$$
\operatorname{Ei}(w)=\gamma+\ln w+\sum_{n=1}^{\infty} \frac{w^{n}}{n n !}<\gamma+\ln w+e^{w} .
$$

For $x \rightarrow \infty, I \sim \mathcal{O}(1)$.

We now consider the vicinity of the resonance, $x \sim 2 c k_{z} / 3 \Omega_{p} k_{y}$. Although $I_{A}$ and $I_{C}$ are divergent logarithmically, the combination $I_{A}-I_{C}$ does not diverge:

$$
I_{A}-I_{C} \sim \mathcal{P} \int_{-k_{z} \delta}^{k_{z} \delta} d t \frac{e^{t}}{t}
$$

where we set $x \sim 2 c k_{z} / 3 \Omega_{p} k_{y}+\delta$. It is easy to show $I_{B}, I_{D} \sim \mathcal{O}(1)$ and therefore, $I$ is order of unity for all $x$. Although $\psi_{p}$ is not strictly a constant, it is smooth in the vicinity of the resonance. Therefore, we can safely assume this to be constant when we discuss the divergence at the resonance.

In summary, from (49), the order of magnitude of $\operatorname{Re} f$ is

$$
\operatorname{Re} f \sim \mathcal{O}\left(\frac{c k_{z}^{2}}{\Omega_{p} k_{y}} \psi_{p}\right) .
$$

It is clear from the equation (50), the imaginary part of $f$ is also of the same order. 


\section{REFERENCES}

Abramowitz, M. \& Stegun, I. A., 1970 Handbook Of Mathematical Functions (New York: Dover)

Artymowicz, P. 1993, ApJ, 419, 155

Bailes, M., Lyne, A. G., \& Shemar, S. L. 1991, Nature, 352, 311

Balbus, S. A., \& Hawley, J. F. 1991, ApJ, 376, 214

D’Angelo, G., Kley, W. \& Henning, T. 2003, ApJ, 586, 540

Fromang, S., Terquem, C., \& Nelson, R. P. 2005, MNRAS, 363, 943

Gammie, C., F. 1996, ApJ, 457, 355

Goldreich, P., \& Tremaine, S. 1979, ApJ, 233, 857

Hayashi, C., Nakazawa, K., \& Nakagawa, Y. 1985, in Protostars and Planets II, ed. Black \& Matthews (Tucson: Univ. Arizona Press)

Haisch, K., E., Lada, E., A., \& Lada, C. J. 2001, ApJ, 553, L153

Inutsuka, S., \& Sano, T., 2005, ApJ, 628, L155

Laughlin, G., Steinacker, A., \& Adams, F., C., 2004, ApJ, 608, 489

Machida, M. N., Matsumoto, T., Tomisaka, K., \& Hanawa, T. 2005, MNRAS, 362, 369

Matsumoto, T., \& Hanawa, T. 2003, ApJ, 583, 269

Meyer-Vernet, N., \& Sicardy, B. 1987, Icarus, 69, 157

Narayan, R., Goldreich, P., \& Goodman, J. 1987, MNRAS, 228, 1

Nelson, R. P. \& Papaloizou, J. C. B. 2004, MNRAS, 350, 849

Press, W. H., Flannery, B. P., Teukolsky, S. A., \& Vetterling, W. T., 1992, Numerical Recipes in Fortran 90: The Art of Scientific Computing, (Cambridge: Cambridge Univ. Press)

Sano, T., Miyama, S. M., Umebayashi, T. \& Nakano, T. 2000, ApJ, 543, 486

Stix, T. H. 1992, Waves in Plasmas, (New York: American Institute of Physics) 
Takeuchi, T. \& Miyama, S. M. 1998, PASJ, 50, 141

Tanaka, H., Takeuchi, T. \& Ward, W. R. 2002, ApJ, 565, 1257

Tanaka, H. \& Ward, W. 2004 ApJ, 602, 388

Terquem, C. 2003, MNRAS, 341, 1157 565, 1257

Ward, W. R. 1986, Icarus, 67, 164 565, 1257

Ward, W. R. 1997, Icarus, 126, 261

Zhang, H. \& Lai, D. 2006, MNRAS, 368, 917 565, 1257 


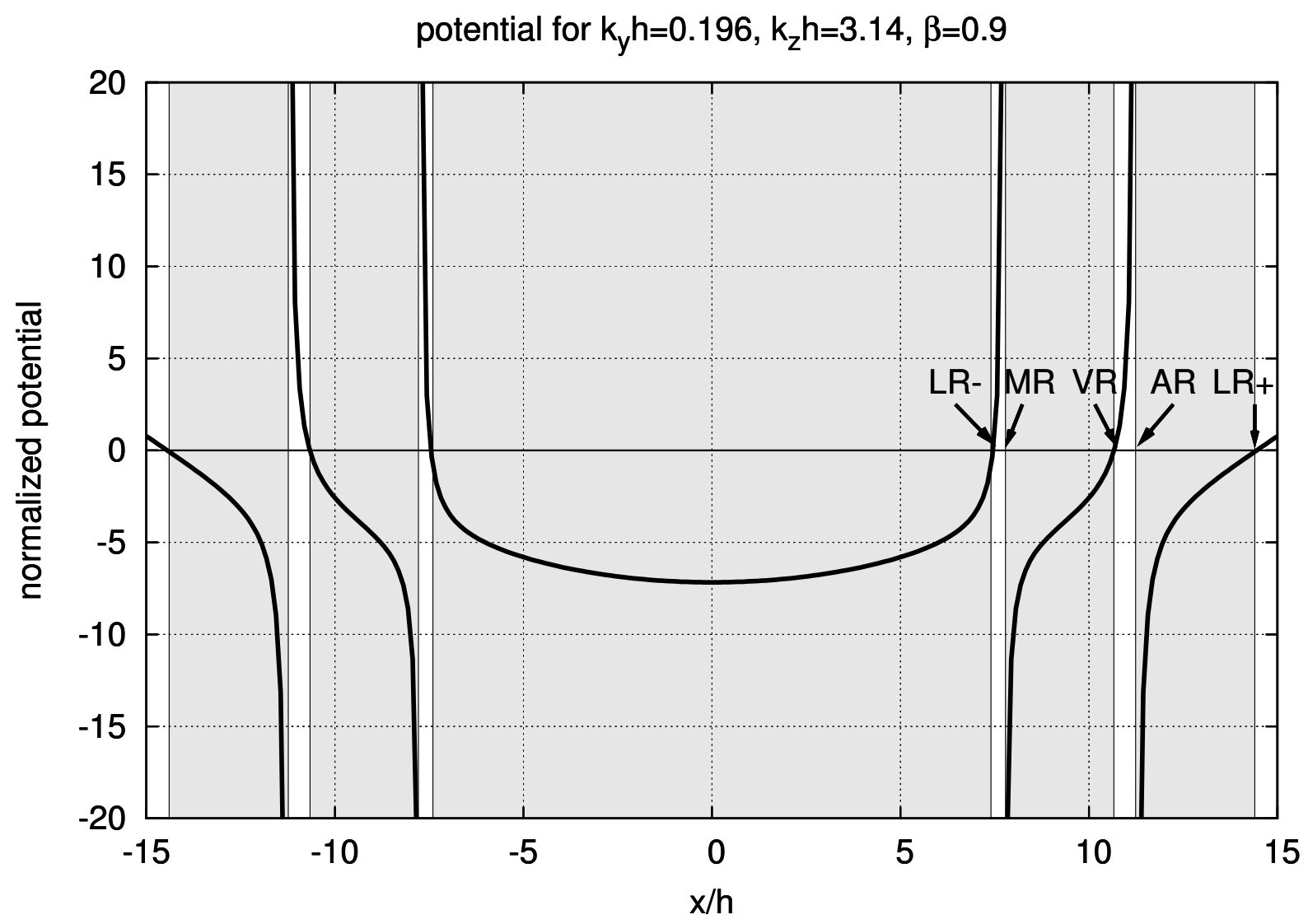

Fig. 1.- Potential $V(x)$ given by equation (32) for $\beta=0.9, k_{y} h=0.196$, and $k_{z} h=3.14$. Resonance positions in the outer disk $(x>0)$ are indicated. LR + and LR- denote Lindblad resonances, AR denotes Alfvén resonance, VR denotes vertical resonance, and MR denotes magnetic resonance. The grey regions correspond to the evanescent regions. Note that regions $|x / h|>15$ are all propagation regions. 


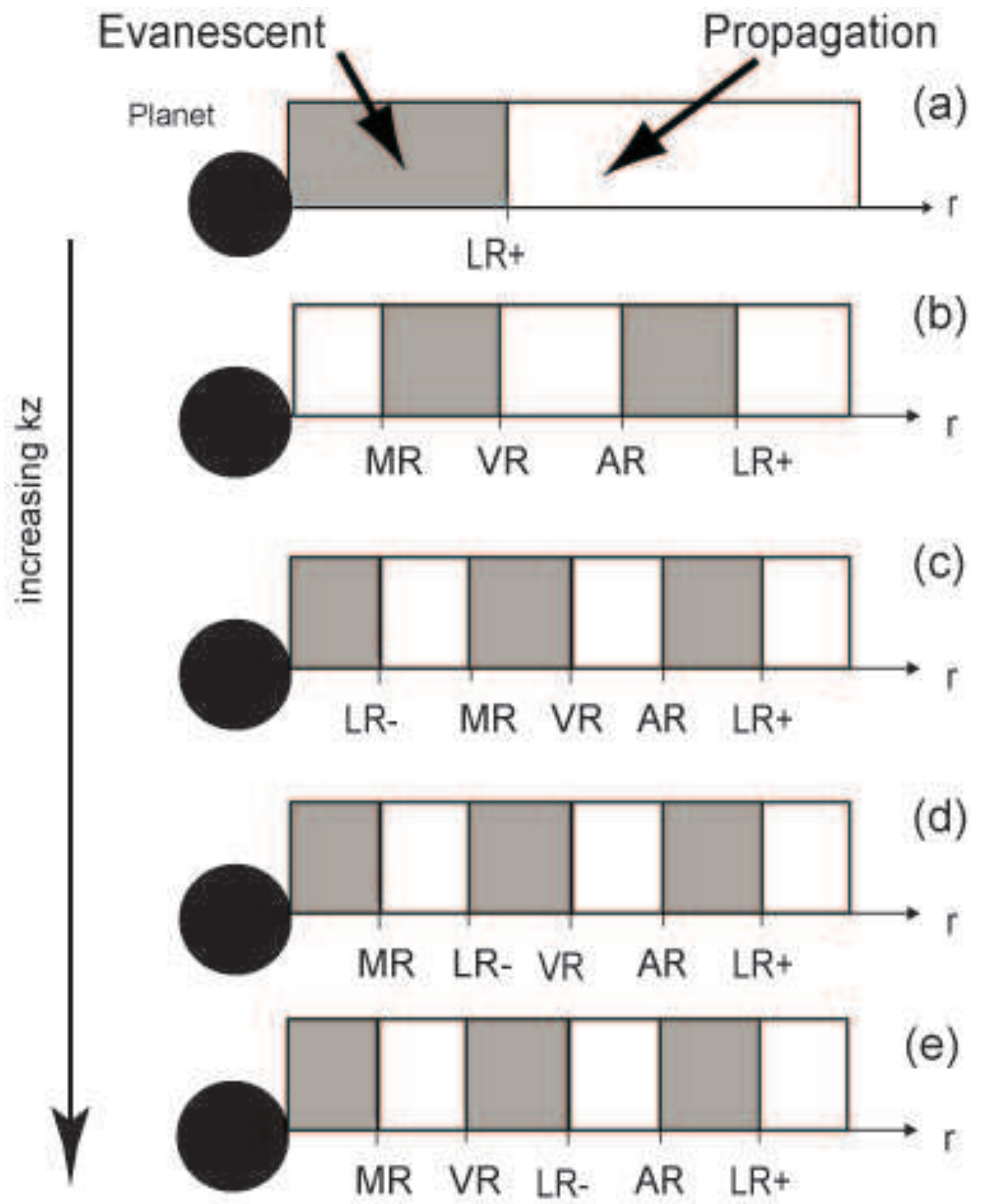

Fig. 2.- Wave propagation property for $\beta<1$ disk. The parameters used in figure 1 corresponds to case (c). 

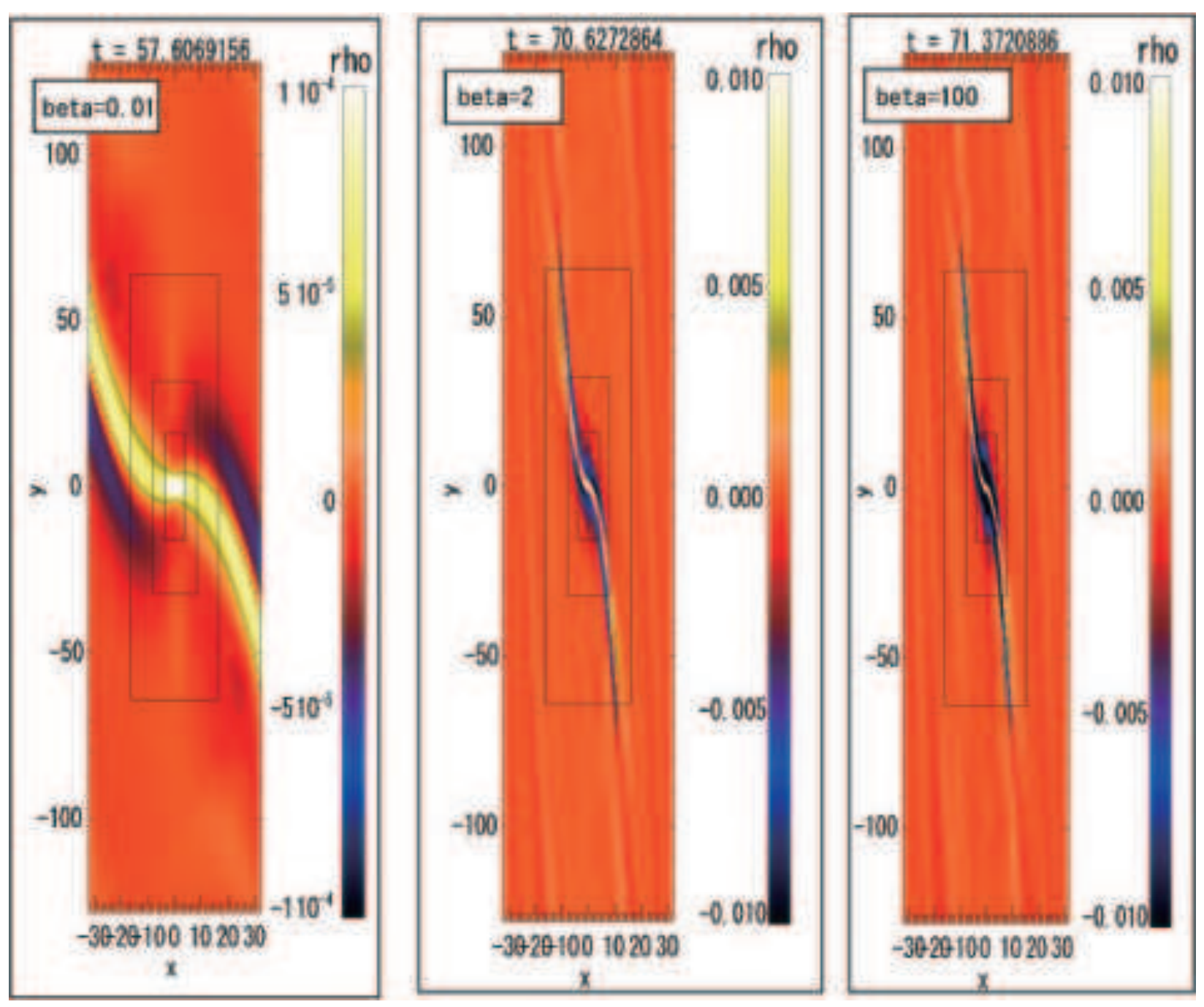

Fig. 3.- Density profile obtained by the two-dimensional numerical calculation. The pattern of density perturbation $\delta \rho / \rho_{0}$ is indicated by false color. It is clear that the stronger the magnetic field, the further the point where waves are excited and the smaller the amplitude. Note that color scales are different for different values of $\beta$. The $x$ - and $y$-axes correspond to the axes of shearing-sheet, normalized by the scale height $c / \Omega_{p}$. The elapsed time $t$ is normalized by the planet's Kepler time $\Omega_{p}^{-1}$. Four different levels of nested grid are superimposed. 


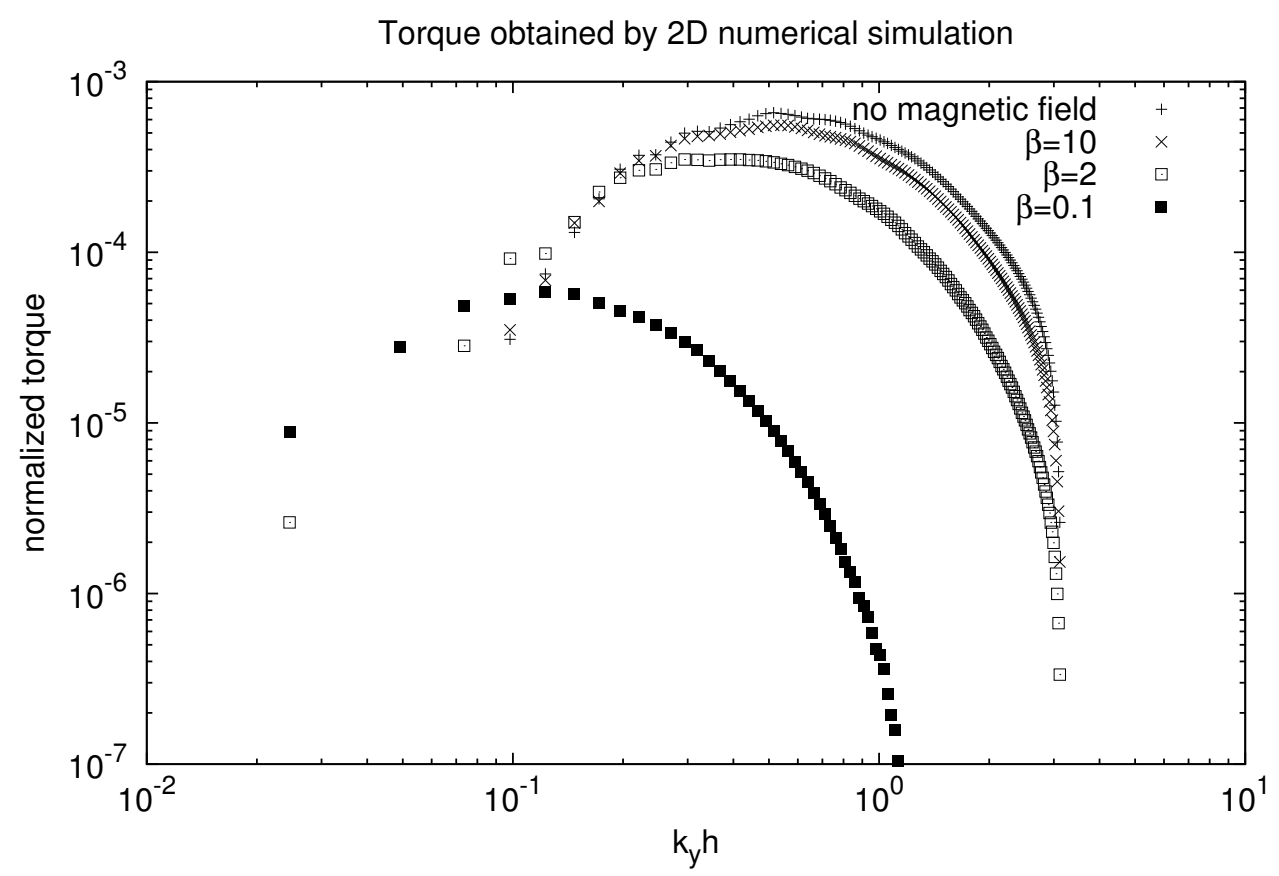

Fig. 4.- The dependence of two-dimensional torque $\left(k_{z}=0\right.$ modes $)$ on the strength of magnetic field obtained by numerical calculations. The models with $\beta=\infty$ (no magnetic field, plus), 10 (cross), 2 (open square), and 0.1 (filled square) are shown. The torque is cut off drastically for models with $\beta<1$. The horizontal axis denotes $k_{y} h$ and the vertical axis denotes normalized torque. 

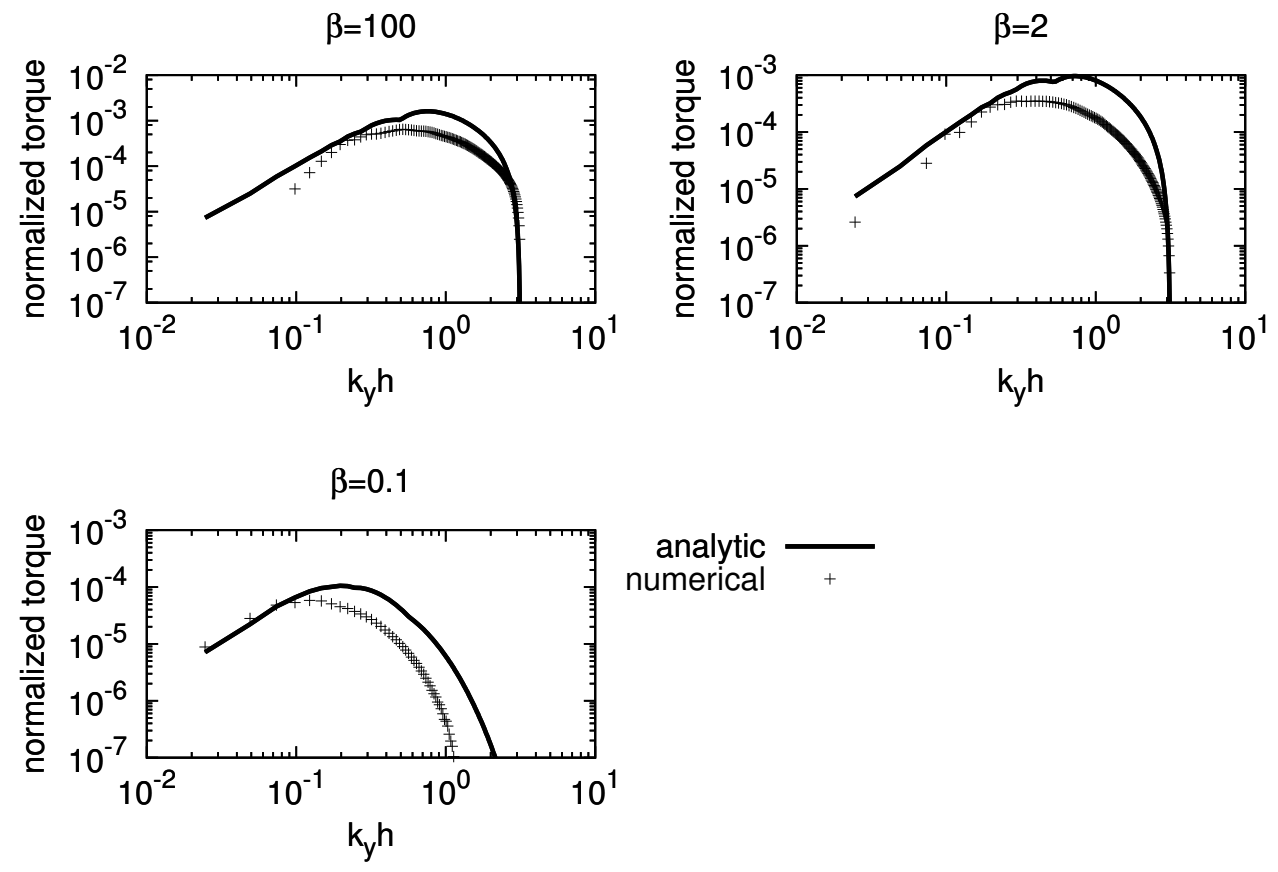

Fig. 5. - Comparison of the torque obtained by the two-dimensional numerical calculation (plus) and the linear analysis (line), the equation (39) for $\beta=100$ (top left), $\beta=2$ (top right), and $\beta=0.1$ (bottom). The horizontal axis denotes the azimuthal mode number and the vertical axis denotes normalized torque. 

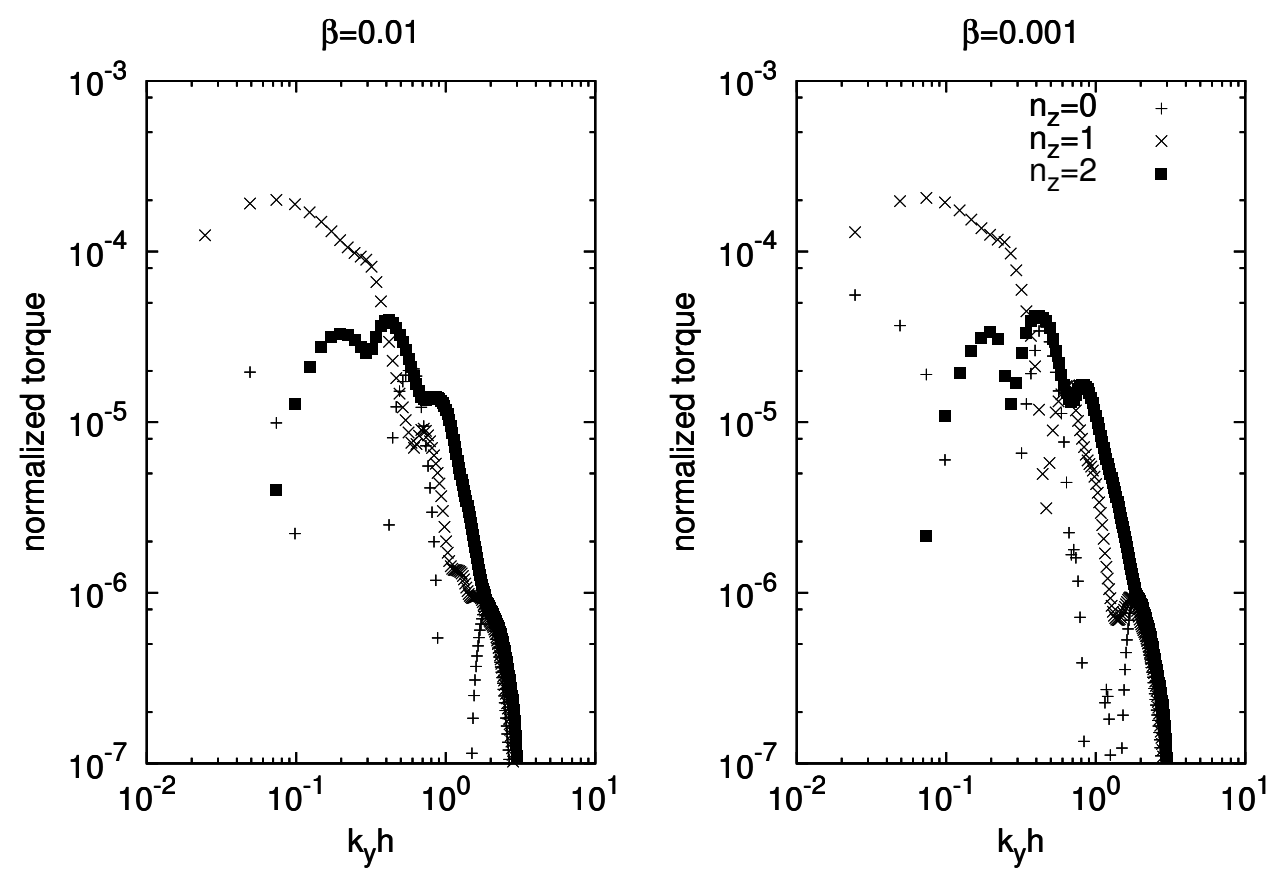

Fig. 6. - $k_{z}$-dependence of the torque obtained by three-dimensional numerical calculations. The left panel shows $\beta=0.01$ and the right $\beta=0.001$. The horizontal axis denotes the azimuthal mode number and the vertical axis denotes normalized torque. Two-dimensional modes are denoted by plus, three-dimensional modes with $n_{z}=1$ by cross, and $n_{z}=2$ by filled square. Three-dimensional modes with $n_{z}=1$ dominate the two-dimensional modes when the magnetic field is sufficiently strong $(\beta \ll 1)$. 
$n_{z}=1$ mode torque, $\beta=0.01$ and $\beta=0.001$

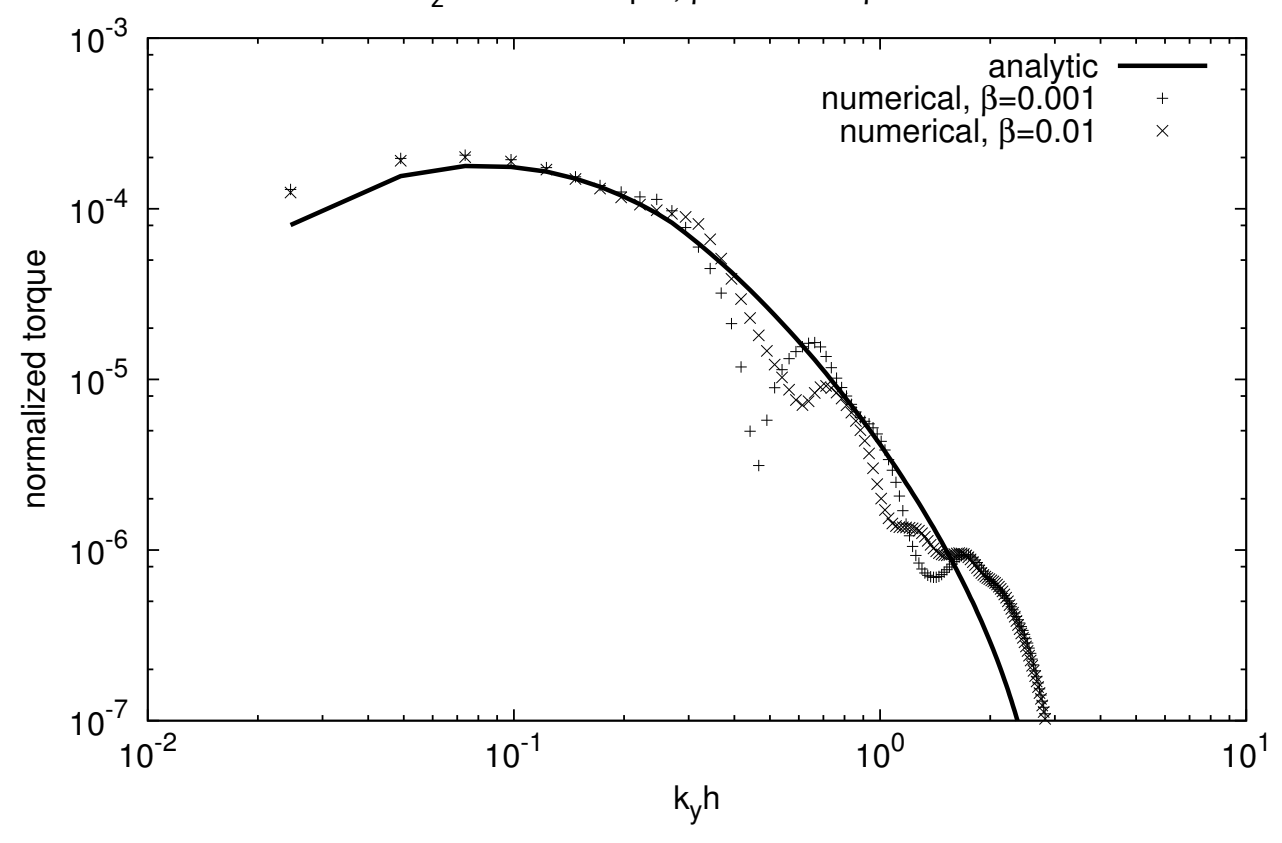

Fig. 7.- The comparison of $n_{z}=1$ mode torque between the analytic formula (line), equation (39), and three-dimensional numerical calculation for $\beta=0.001$ (plus) and $\beta=0.01$ (cross). The horizontal axis shows the azimuthal mode number and the vertical axis shows the normalized torque. 


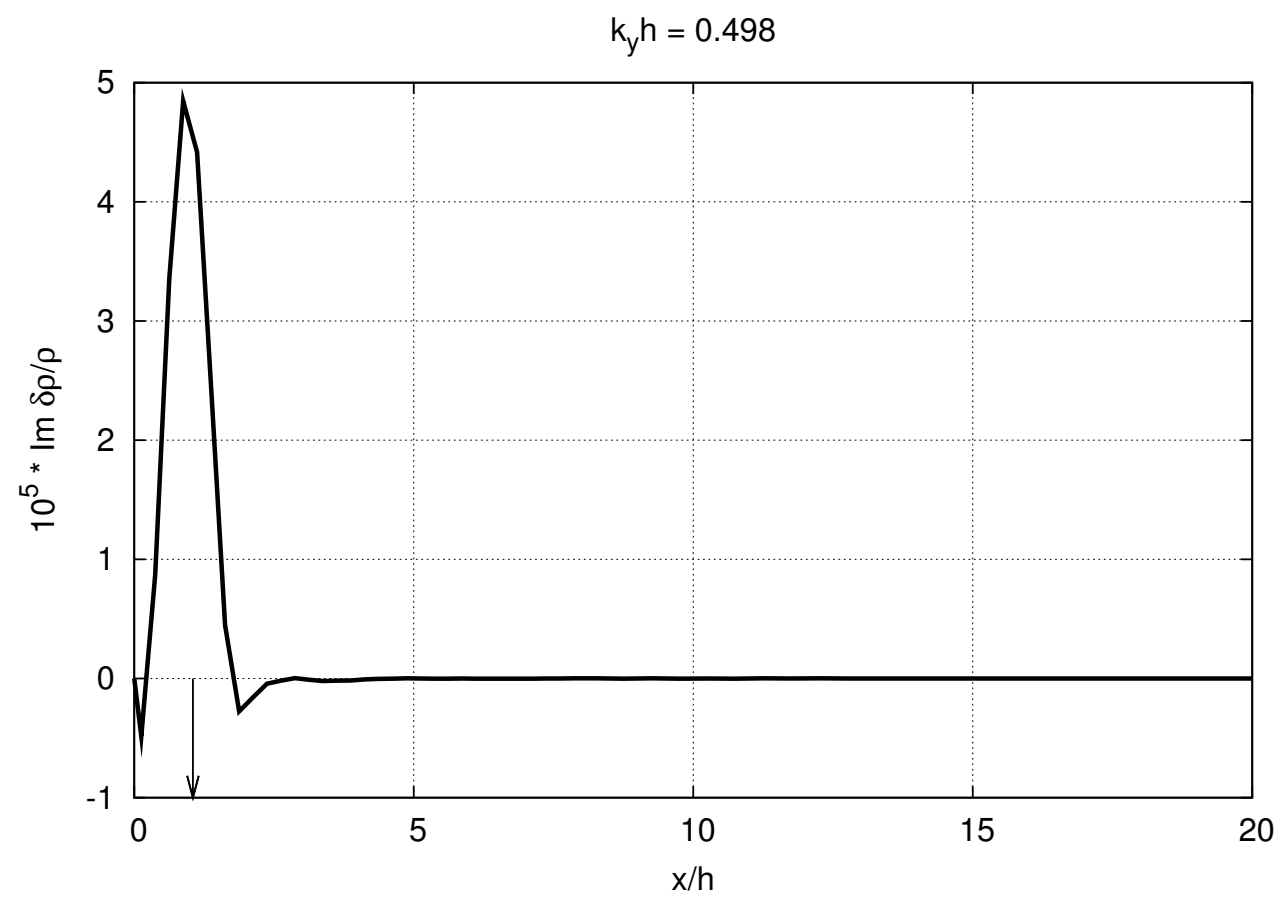

Fig. 8. - The radial profile of $\operatorname{Im}\left(\delta \rho / \rho_{0}\right)$ for the Fourier component $\left(k_{y} h, k_{z} h\right)=$ $(0.498,0.785)$ obtained by $\beta=0.001$ numerical calculation. The profile of the torque on the disk depends on the imaginary part of the density perturbation [see equation (19)]. The horizontal axis shows the radial coordinate and the arrow indicate the position of the magnetic resonances calculated by the linear analysis. 

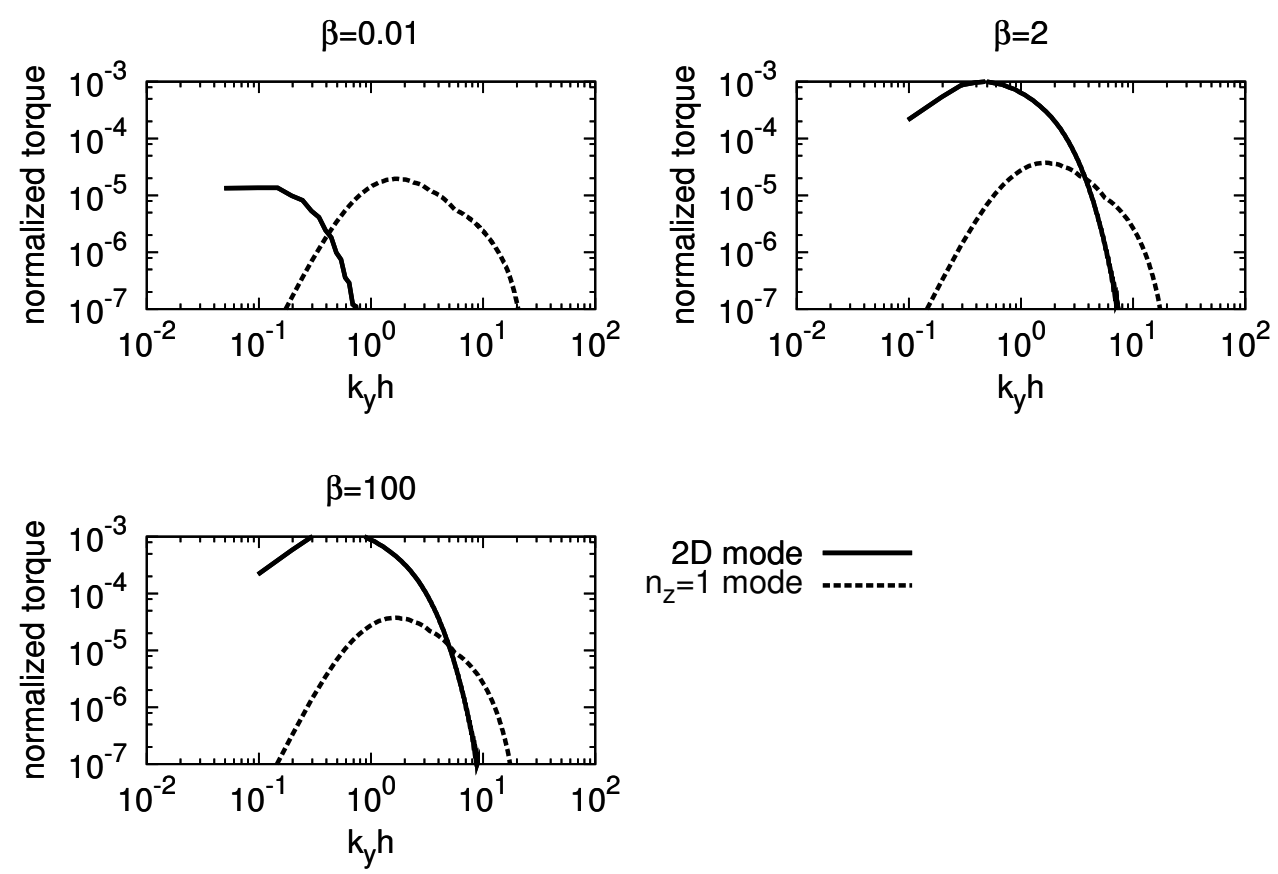

Fig. 9.- Comparison between two-dimensional torque formula (39) and three-dimensional formula (56) for thin disk with $\beta=0.01$ (top left), $\beta=2$ (top right), and $\beta=100$ (bottom). Two-dimensional torque is denoted by the solid line and three-dimensional torque is denoted by the dashed line. The horizontal axis denotes azimuthal mode number and the vertical axis denotes normalized torque. 


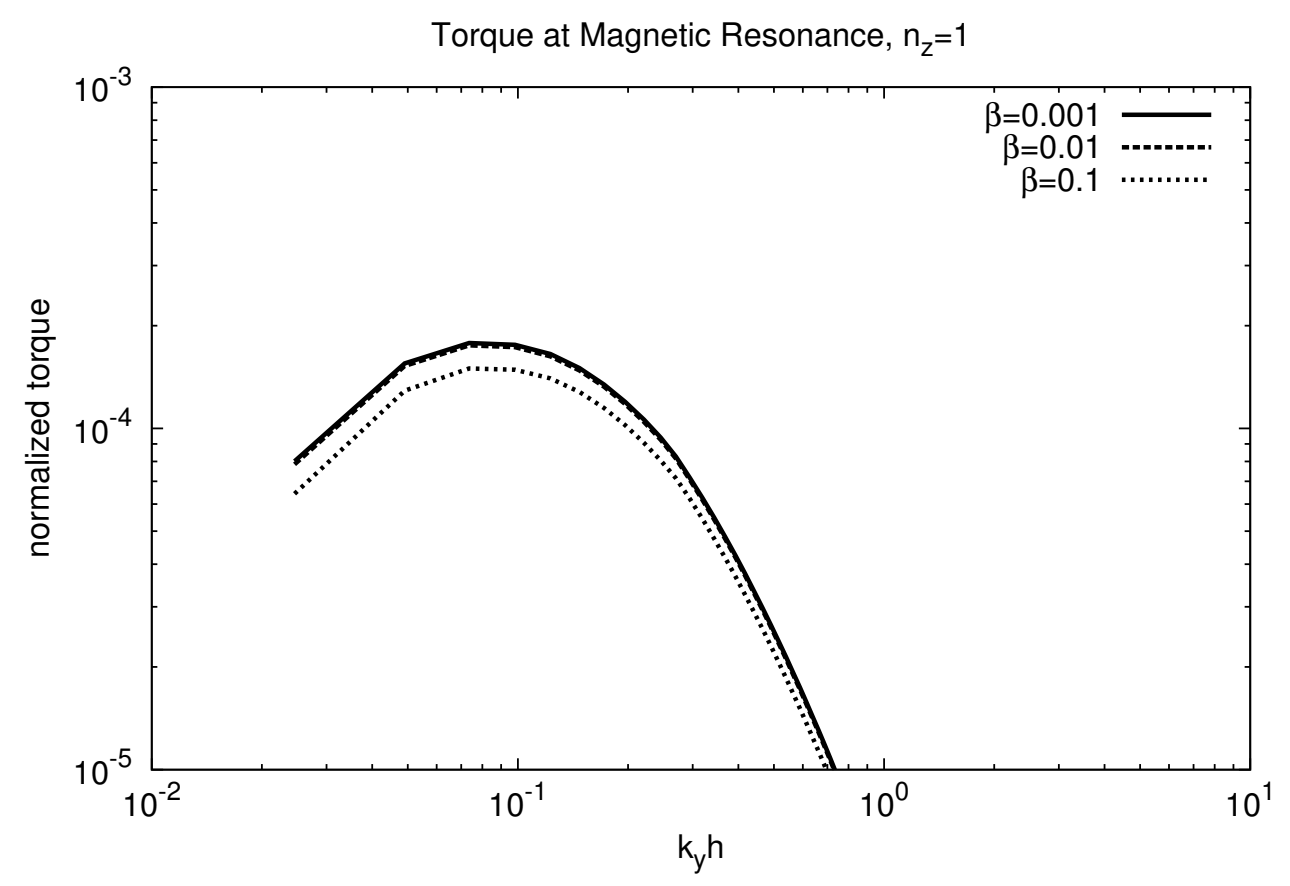

Fig. 10. - Torque at the magnetic resonance obtained by equation (62). Magnetic field strength corresponds to $\beta=0.001$ (solid line), $\beta=0.01$ (dashed line), and $\beta=0.1$ (dotted line). The horizontal axis denotes azimuthal mode number and the vertical axis denotes normalized torque. 